\title{
Detailed investigation into the preparation of graphene oxide by dichromate oxidation
}

\author{
Martin Rosillo-Lopez ${ }^{[a]}$ and Christoph G. Salzmann ${ }^{\star[a]}$
}

\begin{abstract}
Graphene oxide (GO) can be prepared using numerous protocols including the well-known Hummers method that relies on potassium permanganate as the oxidizing agent. Given the widespread use of $\mathrm{GO}$ and its many applications, a better understanding of the mechanisms of chemical oxidation is highly desirable including research into alternative oxidizing agents. Limited work has so far been carried out into using potassium dichromate as an oxidizing agent and the chemical structure of GO prepared by dichromate oxidation remains unclear. Here we present a full structural characterization of GO prepared by dichromate oxidation and its thermal stability. Furthermore, we optimize the previously reported protocol by reducing the reaction time from 5 days to 20 hours and showing that sodium nitrate is not needed in the reaction mixture. Contrary to previous reports, we show that the GO material produced by dichromate oxidation is highly oxidized if thermal annealing is avoided. Compared to traditional GO, larger amounts of carboxylic acid groups are found which constitutes a major advantage for chemical processing and applications in materials chemistry.
\end{abstract}

\section{Introduction}

The chemistry of graphene oxide (GO), the oxidized counterpart of the graphene, and its potential use in applications and devices have been the focus of intense research over the last decade. ${ }^{[1]}$ For instance, GO has been used for desalination and water purification, ${ }^{[2]}$ its antimicrobial properties, ${ }^{[3]}$ membranes, ${ }^{[4]}$ electrocatalysis, ${ }^{[5]}$ composites $^{[6]}$ and ionic sieving. ${ }^{[7]}$ The interest in GO is owed to its hydrophilic nature, multitude of functional groups, and facile and cheap preparation. GO was first synthesized from flake graphite by Brodie in 1859 using the harsh oxidizing conditions created by $\mathrm{KClO}_{3}$ in fuming $\mathrm{HNO}_{3}{ }^{[8]}$ The procedure was modified by Staudenmaier in 1898 by replacing approximately two-thirds of the fuming $\mathrm{HNO}_{3}$ with $\mathrm{H}_{2} \mathrm{SO}_{4}$ and adding the $\mathrm{KClO}_{3}$ to the reaction mixture slowly in aliquots over 4 days. ${ }^{[9]}$ The advantages of the Staudenmaier approach over the Brodie method are three-fold. Firstly, the procedure reduces the risk of explosion as a result of $\mathrm{ClO}_{2}$ gas formation, secondly it increases the overall oxidation degree of $\mathrm{GO}$ and finally, the reaction can be contained to a single reaction vessel. The procedure was further improved by Hummers and Offeman in 1958 by replacing $\mathrm{KClO}_{3}$ and fuming $\mathrm{HNO}_{3}$ with potassium permanganate $\left(\mathrm{KMnO}_{4}\right)$ and potassium nitrate $\left(\mathrm{KNO}_{3}\right)$ in concentrated $\mathrm{H}_{2} \mathrm{SO}_{4}$, thereby significantly reducing the risk of

[a] Dr M. Rosillo-Lopez, Dr C. G. Salzmann

Department of Chemistry

University College London

20 Gordon Street

London WC1H OAJ

UK

E-mail: c.salzmann@ucl.ac.uk

Supporting information for this article is available on the WWW under http://dx.doi.org/10.1002/chem.2018xxxxx explosion and other hazards such as $\mathrm{NO}_{x}$ fumes. ${ }^{[10]}$ It is noteworthy that the original use of a $\mathrm{KMnO}_{4} / \mathrm{H}_{2} \mathrm{SO}_{4}$ mixture to oxidize graphite was reported by Charpy around fifty years before Hummers and Offeman. ${ }^{111]}$

Nowadays, GO is most commonly synthesized via modified Hummers methods of which there are numerous variations. ${ }^{[12]}$ For instance, Chen et. al. have shown that GO can be synthesized in under one hour while using fewer equivalents of $\mathrm{KMnO}_{4}$ than originally reported. ${ }^{[12 a]}$ Alternatively, the 'improved synthesis of graphene oxide' developed by Marcano and co-workers in 2010, which replaces concentrated $\mathrm{H}_{2} \mathrm{SO}_{4}$ with a mixture of 9:1 $\mathrm{H}_{2} \mathrm{SO}_{4}: \mathrm{H}_{3} \mathrm{PO}_{4}$, has also gained popularity. ${ }^{[13]}$ Other novel oxidizing agents such as $\mathrm{K}_{2} \mathrm{FeO}_{4}$ have also seen an increase in interest over the last couple of years, owing to shorter reactions times and the prevention of toxic manganese waste. ${ }^{[14]}$ Similarly, non-wet-chemical approaches towards the synthesis of GO are also being considered such as water electrolytic oxidation which relies on highly reactive radicals produced via the electrolysis of water. ${ }^{[15]}$ Consequently, $\mathrm{KMnO}_{4}$ and other metal-based oxidizing agents can be avoided. However, $\mathrm{H}_{2} \mathrm{SO}_{4}$ is still required for the initial intercalation of graphite.

Chandra and co-workers reported the first preparation of $\mathrm{GO}$ using $\mathrm{K}_{2} \mathrm{Cr}_{2} \mathrm{O}_{7}$, as an alternative to $\mathrm{KMnO}_{4}{ }^{[16]}$ The new $\mathrm{GO}$ was shown to have a significantly more negative zeta potential compared to GO prepared by the Hummers methods. However, limited structural characterizations, in particular a lack of XPS, ${ }^{13} \mathrm{C}$-ssNMR and AFM measurements, has meant that the chemical nature of the new GO material remained elusive. In the few follow-up publications by other groups, ${ }^{[12 b, 17]}$ XPS has been utilized as a characterization method by some. ${ }^{[12 b, 17 a, 17 b]}$ The results indicated that GO prepared by dichromate oxidation, now referred to as DC-GO, is a significantly less oxidized carbon material compared to GO prepared using permanganate (PM$\mathrm{GO}$ ). However, these findings seem to be inconsistent with some of the original observations by Chandra et. al. ${ }^{[16]} \mathrm{DC}$-GO has been shown to display outstanding sorption capacities for lead ions ${ }^{[17 \mathrm{~b}]}$ and has been used for a variety of other applications. ${ }^{[17 a, 17 c, 17 d]}$ Here we aim to investigate the chemical structure of DC-GO in detail and to also optimize the original procedure by Chandra et. al. which requires a reaction time of five days and includes $\mathrm{NaNO}_{3}$ as a reagent. The composition of functional groups on DC-GO is compared with respect to PM-GO and we aim to explain how the in-part contradicting results in the DC-GO literature came about. Finally, the advantages and disadvantages of DC-GO for applications are discussed in comparison with the more traditional PM-GO material.

\section{Results and Discussion}

Improved preparation procedure for DC-GO 
The preparation procedure for DC-GO developed by Chandra et al. uses 7.5 mass equivalents of $\mathrm{K}_{2} \mathrm{Cr}_{2} \mathrm{O}_{7}$ as the oxidizing agent at $20^{\circ} \mathrm{C}$ and a reaction time of 5 days. ${ }^{[16]}$ The aim of our study was to reduce the reaction time to below 24 hours while still obtaining a similar DC-GO material at a comparable yield. In analogy to the modifications to the Hummers method, ${ }^{[12 a]}$ it was also decided to eliminate $\mathrm{NaNO}_{3}$ from the reaction mixture.

The influences of temperature, reaction time and the equivalents of $\mathrm{K}_{2} \mathrm{Cr}_{2} \mathrm{O}_{7}$ on the yield of DC-GO are shown in Figure 1. After a reaction time of 20 hours at $20^{\circ} \mathrm{C}$ and using 7.5 equivalents of $\mathrm{K}_{2} \mathrm{Cr}_{2} \mathrm{O}_{7}$, a quite poor yield of $18 \mathrm{w} \%$ was obtained. The temperature of the reaction was therefore raised to $45^{\circ} \mathrm{C}$ in a next step which resulted in a substantial increase of the yield to $94 \mathrm{wt} \%$ for otherwise identical reaction conditions. This highlights a strong temperature dependence of the oxidation kinetics of graphite with $\mathrm{K}_{2} \mathrm{Cr}_{2} \mathrm{O}_{7}$. As further explorations of the reaction conditions at $45^{\circ} \mathrm{C}$ showed, using 7.5 equivalents of $\mathrm{K}_{2} \mathrm{Cr}_{2} \mathrm{O}_{7}$ and a reaction time of 20 hours actually gives the highest yield. Using fewer than 7.5 equivalents of $\mathrm{K}_{2} \mathrm{Cr}_{2} \mathrm{O}_{7}$ resulted in much lower yields suggesting an insufficient amount of the oxidizing agent. Interestingly, increasing the $\mathrm{K}_{2} \mathrm{Cr}_{2} \mathrm{O}_{7}$ equivalents above 7.5 also resulted in lower yields suggesting over-oxidation and hence decomposition of the DC-GO product material in the reaction mixture. In terms of the reaction times, very low yields were obtained for reaction times up to 8 hours which was followed by a massive increase in yield for 20 hours and a subsequent decrease for 40 hours again suggesting over-oxidation of the material upon prolonged oxidative treatment.

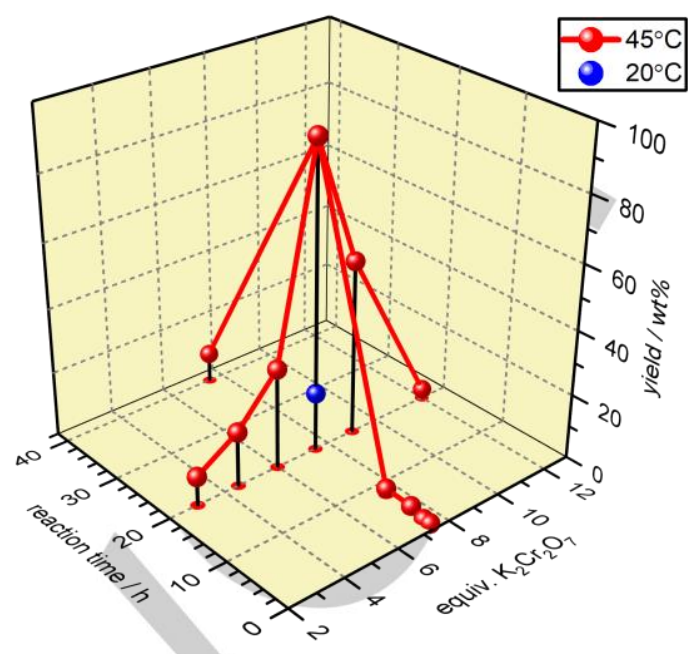

Figure 1. Influences of temperature, reaction time and mass equivalents of $\mathrm{K}_{2} \mathrm{Cr}_{2} \mathrm{O}_{7}$ with respect to the graphite starting material on the yield of DC-GO.

\section{Structural characterization of DC-GO and comparisons with other GO materials}

The XPS and ${ }^{13} \mathrm{C}$ ssNMR spectra of the DC-GO material prepared with the optimized reaction conditions as well as the corresponding data for DC-GO made following the procedure by Chandra et al. are shown in Figure 2(a,d) and $(b, e)$, respectively. For comparison, the XPS and ${ }^{13} \mathrm{C}$ ssNMR spectra of PM-GO synthesized using the protocol by Chen et. al. are also shown in Figure 2(c,f).
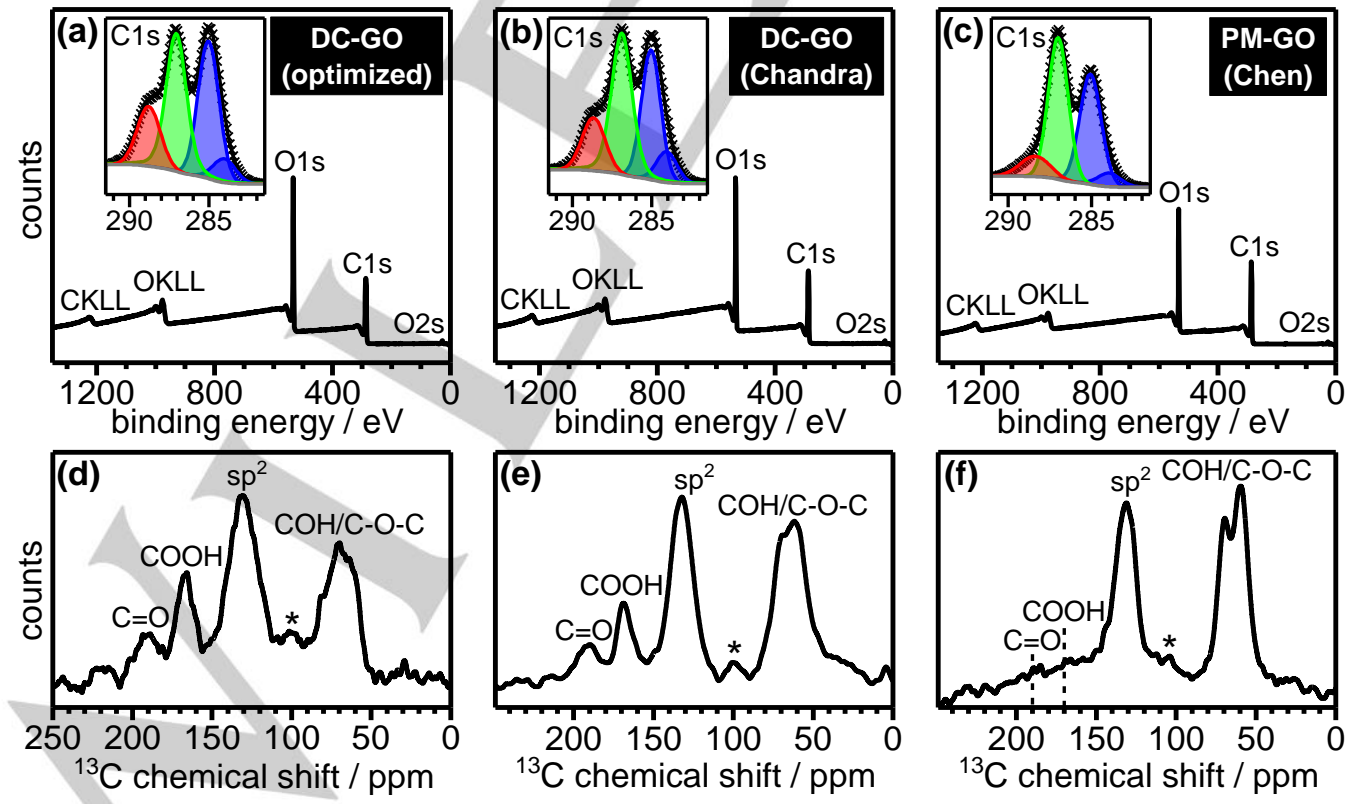

Figure 2. XPS survey spectra and C1s region (insets) of (a) DC-GO (optimized), (b) DC-GO (Chandra) and (c) PM-GO (Chen). In the insets, the crosses represent the experimental data whereas the grey, blue, green, red and black lines are the Shirley background functions, $C(0), C(I)$ and $C(I I I)$ fitted peaks and peak sum, respectively. ${ }^{13} \mathrm{C}$ ssNMR spectra of (d) DC-GO (optimized), (e) DC-GO (Chandra) and (f) PM-GO (Chen). The peaks marked with asterisks are typically assigned to lactol groups. ${ }^{[18]}$ 
The XPS survey spectra of the three carbon materials show carbon and oxygen as the only elements present illustrating the purities of the three materials. The C1s region of the two DC-GO materials are remarkably similar and show a prominent presence of C(III) species as indicated by the fitted peak in red at $\sim 289 \mathrm{eV}$ which is typically attributed to carboxylic acids. ${ }^{[19]}$ The areas of the C(III) peaks were found to be $16-18 \%$ of the overall $\mathrm{C} 1 \mathrm{~s}$ features for both DC-GO samples. The fitted peak in green is assigned to $\mathrm{C}$ (I) functional groups such as hydroxyls and epoxides, and the average $\mathrm{C}$ (III) / C(I) peak area ratio was found to be $0.41 \pm 0.02$ for both samples. It is noteworthy, however, that both peaks are expected to have small contributions from C(II) species such as ketones which cannot be accurately determined due to the somewhat subjective nature of the peak fitting process. The $\mathrm{C} 1 \mathrm{~s}$ spectrum of PM-GO (Chen), which is representative of conventional GO prepared by permanganate oxidation, shows a smaller C(III) peak area $(10-13 \%$ of the overall feature) compared with the DC-GO materials. Similarly, the C(III) / C(I) peak area ratio was calculated as $0.25 \pm 0.02$ which is significantly smaller than the $0.41 \pm 0.02$ obtained for DC-GO indicating a preference for C(III) species over other oxidized carbon in the DCGO materials. Finally, in all C1s spectra, the two fitted peaks in blue are due to the presence of unoxidized carbon. ${ }^{[20]}$

Consistent with the XPS data, the ${ }^{13} \mathrm{C}$ ssNMR spectra of DCGO (optimized) and DC-GO (Chandra) in Figure 2(d,e) show more intense peaks at $\sim 170$ and $\sim 190$ ppm compared with PMGO (Chen) in Figure 2(f) indicating larger amounts of carboxylic acids and ketones / aldehydes, respectively. ${ }^{[18,21]}$ In fact, the $\mathrm{COOH}$ and $\mathrm{C}=\mathrm{O}$ groups are barely noticeable in the ${ }^{13} \mathrm{C}$ ssNMR spectra of PM-GO (Chen), whilst the graphenic $\mathrm{sp}^{2}$ carbon peak centered at $\sim 130 \mathrm{ppm}$ and the hydroxyl / epoxide peaks at 70 / 60 ppm are very prominent. It is noteworthy, that the peaks marked with asterisks at $\sim 100 \mathrm{ppm}$ are typically assigned to lactol groups. ${ }^{[18]}$

The AFM image of DC-GO (optimized) in Figure 3(a) shows that the material is of nanoscale dimensions. Figure $3(b)$ shows an analysis of the flake heights using data from this figure as well as Figure S1. The average height of the flakes was found to be $1.8 \pm 0.8 \mathrm{~nm}$ which suggests $\sim 1-3$ oxidized carbon layers. ${ }^{[22]}$ However, it is apparent from the AFM image and the line profile in Figure 3(c) that smaller adsorbed fragments, previously described as 'dome-like' features, exist on some of the flakes. ${ }^{\text {[22a, }}$ 23] Lastly, analysis of the lateral size distribution of the flakes shown in Figure 3(d) indicates a typical flake diameter around 20 $-30 \mathrm{~nm}$. In contrast to this, the AFM image of PM-GO (Chen) in Figure S2 indicates the presence of much larger flakes up to the micrometer range. The comparatively small flakes of DC-GO are consistent with its relatively large number of carboxylic acid groups which must be located at the edges of the flakes. The same was also found to be the case for DC-GO (Chandra) as shown in Figure S3.
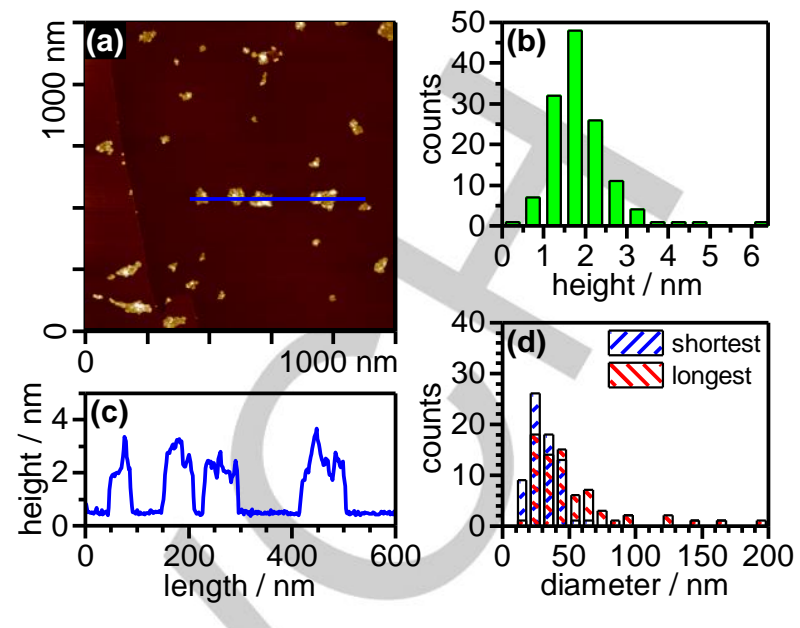

Figure 3. (a) AFM image of DC-GO (optimized) spin coated onto a HOPG substrate, (b) height distribution, (c) line profile as indicated by the blue line in (a), and (d) diameter distribution determined by measuring the shortest and longest distances across individual DC-GO flakes.

The FT-IR and Raman spectra of DC-GO (optimized), DC-GO (Chandra) and PM-GO (Chen) are shown in Figure 4. Overall, the FT-IR and Raman spectra of the three materials are very similar. In the FT-IR spectra, the broad feature centered at $\sim 3300 \mathrm{~cm}^{-1}$ can be assigned to $\mathrm{O}-\mathrm{H}$ stretching from carboxylic acids and water. ${ }^{[24]}$ The carbonyl stretch at $\sim 1715 \mathrm{~cm}^{-1}$ can also be attributed to carboxylic acids as well as other carbonyl functionality such as ketones. ${ }^{[25]}$ Interestingly, there are weak shoulders present adjacent to the carbonyl peak at $\sim 1820 \mathrm{~cm}^{-1}$ for all three samples. Recently, we have shown that these features can be attributed to carboxylic anhydrides. ${ }^{[23,26]}$ The features observed at $\sim 1380$, $\sim 1200$ and $\sim 1050 \mathrm{~cm}^{-1}$ are typically assigned to $\mathrm{O}-\mathrm{H}$ bending and C-O stretching due to the presence of hydroxyl and epoxide groups as well as carboxyls. ${ }^{[27]}$ Lastly, the peak at $\sim 1620 \mathrm{~cm}^{-1}$, which has been the result of some controversy, ${ }^{[28]}$ can be ascribed to the bending mode of water molecules as well as the $\mathrm{C}=\mathrm{C}$ stretching. The Raman spectra of the two DC-GO materials and PM-GO (Chen) show the characteristic $G\left(\sim 1600 \mathrm{~cm}^{-1}\right)$ and D $\left(\sim 1350 \mathrm{~cm}^{-1}\right)$ bands which are observed in all oxidized graphenic materials. These are a result of the in-plane stretching mode of $\mathrm{sp}^{2}$ carbon and the disorder-induced mode, respectively. ${ }^{[29]}$ 

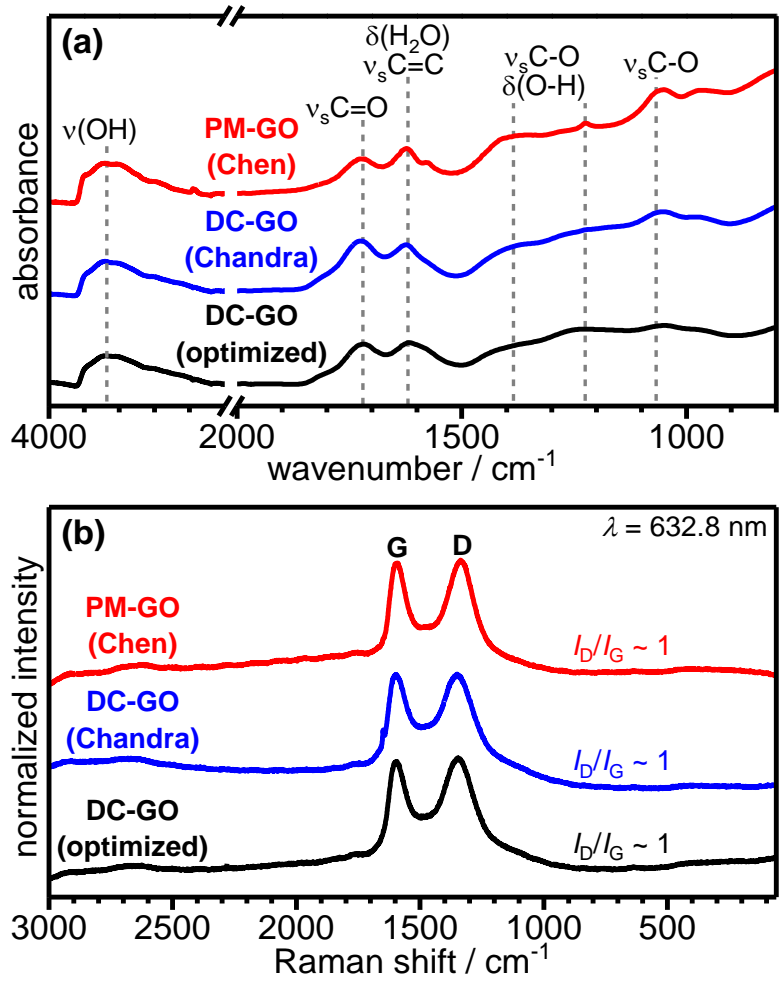

Figure 4. (a) FT-IR and (b) Raman spectra of DC-GO (optimized), DC-GO (Chandra) and PM-GO (Chen).

Finally, in-situ mass spectrometry was employed to elucidate the nature of desorbing gaseous species when DC-GO (optimized) undergoes thermal treatment under high-vacuum conditions starting from room temperature to $900^{\circ} \mathrm{C}$. The mass spectrometry data shown in Figure 5 is consistent with the rapid thermal exfoliation that is typical for GO materials. ${ }^{[30]}$ Below $\sim 120^{\circ} \mathrm{C}$ physisorbed water is removed which is later accompanied by the concerted evolution of $\mathrm{H}_{2} \mathrm{O}, \mathrm{CO}$ and $\mathrm{CO}_{2}$ between 200 and $250^{\circ} \mathrm{C}$. This simultaneous gas release constitutes the removal of most of

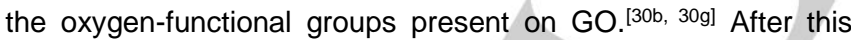
exfoliation step, the desorption of gases slows down and more stable oxo-groups are removed slowly upon further heating. ${ }^{[27 b, 30 \mathrm{~b}}$ 30g, 31] The very similar thermal-desorption pattern of PM-GO (Chen) is shown in the SI of ref. [26].

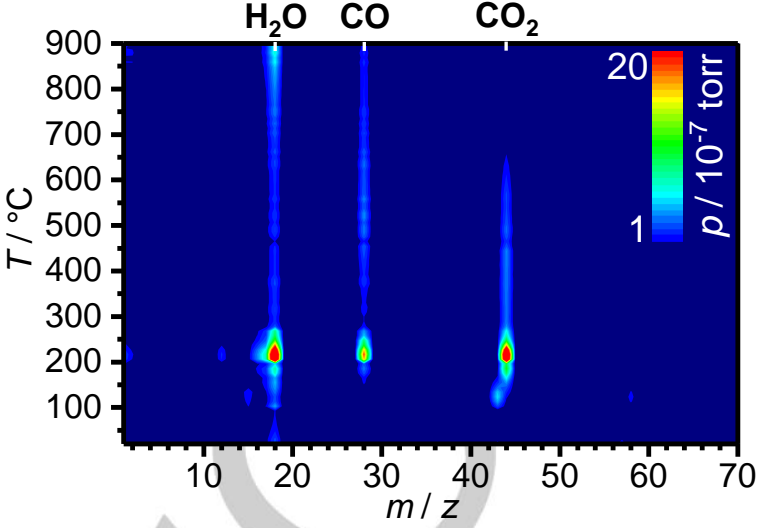

Figure 5. Mass spectrometry data of DC-GO (optimized) recorded upon heating under high-vacuum conditions.

\section{Mechanism of DC-GO formation}

To gain more detailed insights into the chemical mechanism of the DC-GO formation from the graphite starting material, the various samples obtained from the optimization of the reaction conditions were analyzed with XPS (cf. Figures S4-6). First, the $(C(I I I)+C(I)) / C(0)$ peak area ratios were calculated, which are thought to be a measure for the extent of oxidation. As can be seen in Figure $6(\mathrm{a})$, the optimized conditions $\left(45^{\circ} \mathrm{C}, 20\right.$ hours and 7.5 equivalents of $\mathrm{K}_{2} \mathrm{Cr}_{2} \mathrm{O}_{7}$ ) correspond to the most oxidized DC$\mathrm{GO}$ material. Smaller amounts of $\mathrm{K}_{2} \mathrm{Cr}_{2} \mathrm{O}_{7}$ in the reaction mixture appear to limit the oxidation of graphite whereas larger amounts seem to decompose the more oxidized material leaving small amounts of less-oxidized DC-GO behind. In ref. [12b], the preparation procedure of Chandra et al. ${ }^{[16]}$ was adapted by using only using 4 equivalents of $\mathrm{Cr}_{2} \mathrm{O}_{7}{ }^{2-}$. On the basis of XPS measurements it was found that the product material did not show significant oxidation. Considering the data shown in Figure 6(a) and that a reaction temperature of $20^{\circ} \mathrm{C}$ was used in ref. [12b], it is now clear why their DC-GO material showed a low degree of oxidation. 

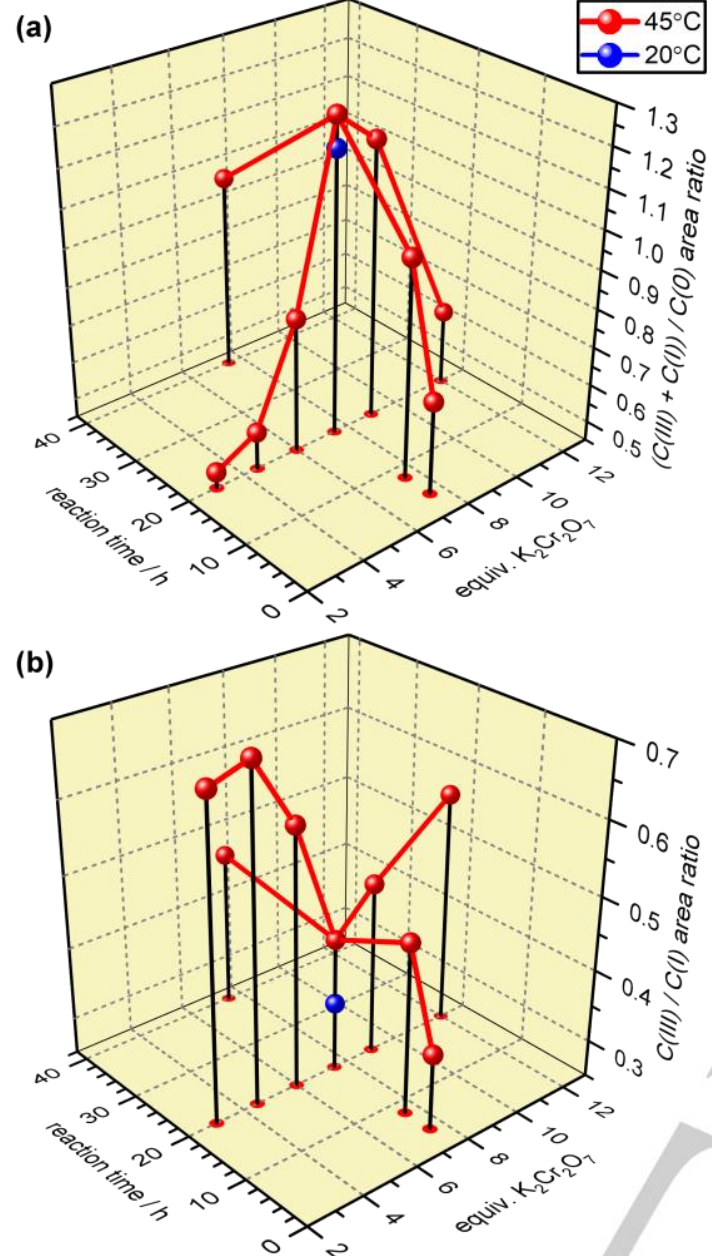

Figure 6. Detailed analysis of the C1s region in the XPS spectra of the various samples prepared for optimizing the yield of the DC-GO (cf. Figure 1). (a) (C(III) $+\mathrm{C}(\mathrm{I})) / \mathrm{C}(0)$ peak area ratios reflecting the extent of oxidation. (b) $\mathrm{C}$ (III) / C(I) peak area ratios highlighting the relative amounts of carboxylic acids with respect to $\mathrm{C}(\mathrm{I})$ functional groups. All peak area ratios are shown as a function of the reaction time and the equivalents $\mathrm{K}_{2} \mathrm{Cr}_{2} \mathrm{O}_{7}$ for the reactions at $20^{\circ} \mathrm{C}$ (blue spheres) and at $45^{\circ} \mathrm{C}$ (red spheres)

Using 7.5 equivalents of $\mathrm{K}_{2} \mathrm{Cr}_{2} \mathrm{O}_{7}$, the extent of oxidation increases during the first 20 hours after which preferential decomposition of the more oxidized material is observed. According to this analysis, the DC-GO (optimized) material is slightly less oxidized compared to PM-GO (Chen) which displays a $(\mathrm{C}(\mathrm{III})+\mathrm{C}(\mathrm{I})) / \mathrm{C}(0)$ peak area ratio of 1.31 .

Also, the extent of oxidation of the DC-GO material obtained at $20^{\circ} \mathrm{C}$ but otherwise optimal conditions is only slightly smaller compared to the material obtained at $45^{\circ} \mathrm{C}$. This is interesting because the yield after the $20^{\circ} \mathrm{C}$ reaction was significantly lower compared to the fully optimized conditions (cf. Figure 1). The difference between these two samples must therefore lie in the relative abundances of the various functional groups.

In a next step, the $\mathrm{C}$ (III) / C(I) peak area ratio is taken as a measure for the amount of carboxylic acid groups with respect to the $\mathrm{C}(\mathrm{I})$ functional groups. As can be seen in Figure 6(b), the DC-
GO sample obtained after heating at $20^{\circ} \mathrm{C}$ contains significantly less carboxylic acid groups compared to the sample obtained from the optimized conditions at $45^{\circ} \mathrm{C}$. Increasing the temperature from 20 to $45^{\circ} \mathrm{C}$ at otherwise identical reaction conditions therefore converts a large fraction of less-oxidized functional groups to carboxylic acid groups.

For 7.5 equivalents $\mathrm{K}_{2} \mathrm{Cr}_{2} \mathrm{O}_{7}$, the $\mathrm{C}$ (III) / C(I) peak area ratio is small for short reaction times but it settles at a more or less constant value after 8 hours. This illustrates the fact that $\mathrm{C}(\mathrm{I})$ functional groups must form first as graphite is oxidized.

It is also interesting to note that for a given reaction time of 20 hours, the DC-GO sample obtained with the highest yield at 7.5 equivalents $\mathrm{K}_{2} \mathrm{Cr}_{2} \mathrm{O}_{7}$ shows the lowest $\mathrm{C}$ (III) / C(I) peak area ratio. This means that while the other samples for this reaction time contain overall less functional groups, the relative fraction of carboxylic acid groups is higher. This could be a reflection of the fact that it is easier to form $\mathrm{C}$ (III) from $\mathrm{C}(\mathrm{I})$ species than it is to perform the first oxidation step starting from $\mathrm{C}(0)$.

Considering that PM-GO (Chen) shows a C(III) / C(I) peak area ratio of 0.26 , it is very clear that all the prepared DC-GO materials are chemically very different compared to PM-GO. ${ }^{[32]}$ This illustrates overall that the oxidation of graphite with $\mathrm{KMnO}_{4}$ or $\mathrm{K}_{2} \mathrm{Cr}_{2} \mathrm{O}_{7}$ proceed via very different chemical mechanisms.

\section{Problems in handling DC-GO due to its thermal instability}

Sitko et. al. isolated DC-GO (Chandra) by drying it at $100^{\circ} \mathrm{C}$ for an undefined duration of time. ${ }^{[17 a, 17 b]}$ On the basis of XPS measurements they found a significantly less oxidized material than reported by us here. In Figure 7 we show that DC-GO is not stable at $100^{\circ} \mathrm{C}$ over prolonged periods of time by placing a sample in an oven at $100^{\circ} \mathrm{C}$ and measuring the changes in functional group composition over time using XPS. There is an obvious decrease in the number of oxo-groups as time progresses as can be seen by the decreasing areas of the $\mathrm{C}$ (III) and $\mathrm{C}(\mathrm{I})$ peaks with respect to the unoxidized carbon peak. In fact, after just 16 hours the $(\mathrm{C}(\mathrm{III})+\mathrm{C}(\mathrm{I})) / \mathrm{C}(0)$ peak area ratio decreased from $\sim 1.39$ to $\sim 0.89$ and a mass loss of $\sim 16 \%$ was noted. It is noteworthy that if the DC-GO has been isolated via the boiling of water from a dispersion of DC-GO in refs [17a, 17b] then the reduction of the material may have been even more pronounced. In summary, it is clear that great caution must be taken during the workup and isolation of DC-GO materials. Freeze-drying of the product dispersions, as carried out by us here, seems to be the optimal way to isolate DC-GO. 

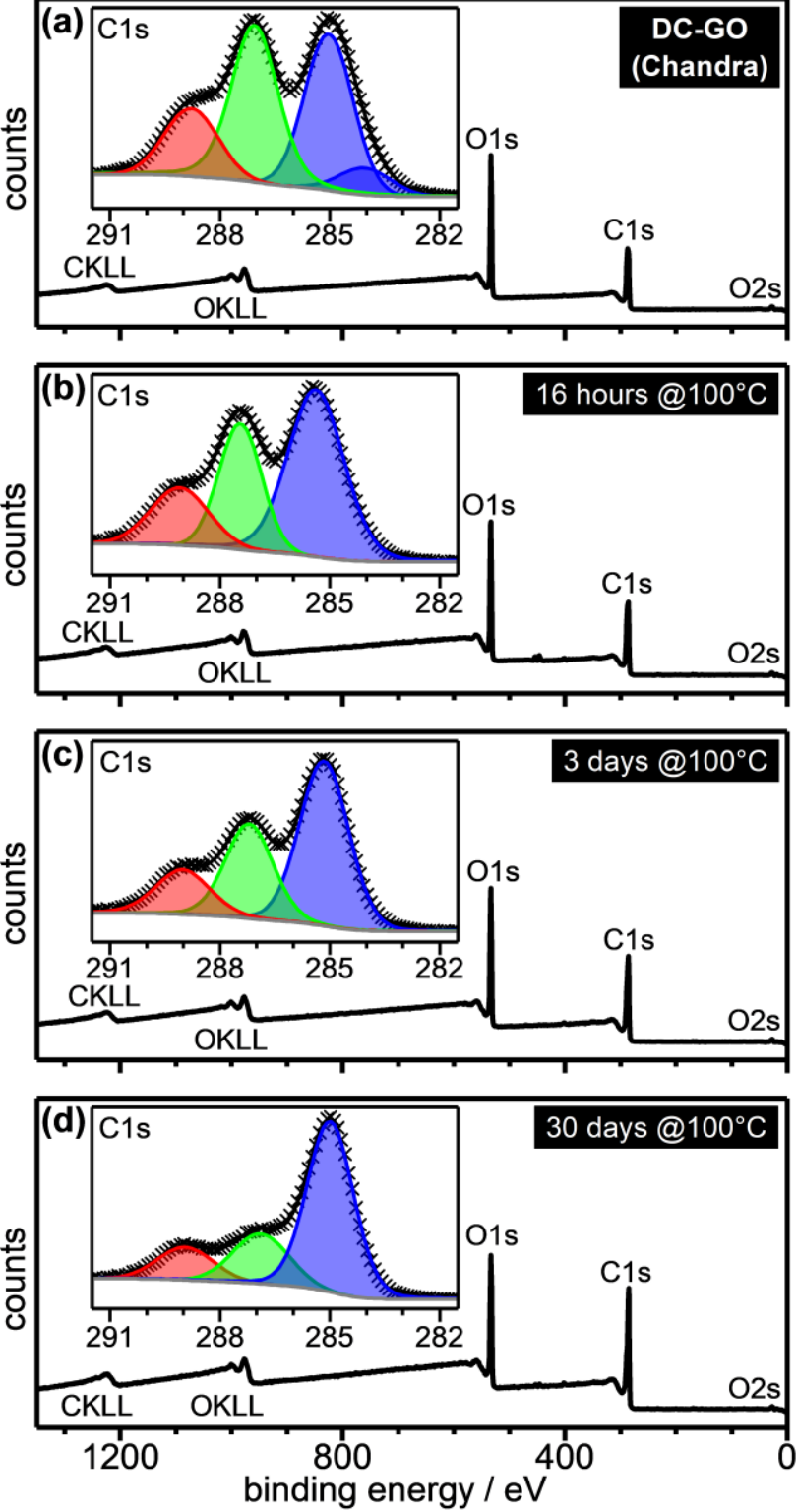

Figure 7. XPS survey spectra and C1s region (insets) of DC-GO (Chandra) after heating at $100^{\circ} \mathrm{C}$ in air for (a) 0 hours, (b) 16 hours, (c) 3 days and (d) 30 days. In the insets, the crosses represent the experimental data whereas the grey, blue, green, red and black lines are the Shirley background functions, C(0), C(I) and C(III) fitted peaks and peak sum, respectively.

\section{Conclusions}

The original protocol developed by Chandra et. al. was optimized by reducing the reaction time from 5 days to 20 hours and removing $\mathrm{NaNO}_{3}$ from the synthesis. Overall, DC-GO was found to be heavily oxidized and more carboxylated than conventional $\mathrm{GO}^{[32-33]}$ which can constitute an advantage towards the chemical processing of GO. In fact, the diverse chemistry of carboxylic acid groups has led to significant interest in carboxylated graphene nanomaterials in recent years. ${ }^{[23,26,34]}$ Consequently, the $\mathrm{COOH}$ groups of GO have been exploited in many chemical functionalization procedures to prepare a multitude of composite materials and to use chemically modified GO in devices. ${ }^{[34 d, 34 e,}$ ${ }^{34 g-n]}$ In this sense, DC-GO provides a unique opportunity for future work in this area due to its high content of carboxylic acid groups and the cheap graphite starting material.

\section{Acknowledgements}

We thank the Royal Society for funding (UF150665), K. Holt for access to ATR-FTIR instrumentation, A. Aliev for recording solidstate NMR spectra, and J. A. Darr and A. Groves for preliminary BET measurements. This project has received funding from the European Research Council (ERC) under the European Union's Horizon 2020 research and innovation programme (grant agreement No 725271)

Keywords: carbon $\bullet$ graphene oxide $\bullet$ oxidation $\bullet$ nanoparticles $\bullet$ chromates

[1] a) V. Georgakilas, J. N. Tiwari, K. C. Kemp, J. A. Perman, A. B. Bourlinos, K. S. Kim, R. Zboril, Chem. Rev. 2016, 116, 5464-5519; b) D. R. Dreyer, S. Park, C. W. Bielawski, R. S. Ruoff, Chem. Soc. Rev. 2010, 39, 228-240; c) L. G. Guex, B. Sacchi, K. F. Peuvot, R. L. Andersson, A. M. Pourrahimi, V. Strom, S. Farris, R. T. Olsson, Nanoscale 2017, 9, 9562-9571.

[2] a) H. M. Hegab, L. Zou, J. Membr. Sci. 2015, 484, 95-106; b) W. Peng, H. Li, Y. Liu, S. Song, J. Mol. Liq. 2017, 230, 496504; c) M. Rosillo-Lopez, C. G. Salzmann, RSC Adv. 2018, 8, 11043-11050.

[3] F. Perreault, A. F. de Faria, S. Nejati, M. Elimelech, Acs Nano 2015, 9, 7226-7236.

[4] Q. Yang, Y. Su, C. Chi, C. T. Cherian, K. Huang, V. G. Kravets, F. C. Wang, J. C. Zhang, A. Pratt, A. N. Grigorenko, F. Guinea, A. K. Geim, R. R. Nair, Nat. Mat. 2017, 16, 1198

[5] K. Qu, Y. Zheng, S. Dai, S. Z. Qiao, Nano Energy 2016, 19, 373-381.

[6] Z. U. Khan, A. Kausar, H. Ullah, Polym. Plast. Technol. Eng. 2016, 55, 559-581.

[7] J. Abraham, K. S. Vasu, C. D. Williams, K. Gopinadhan, Y. Su, C. T. Cherian, J. Dix, E. Prestat, S. J. Haigh, I. V. Grigorieva, P. Carbone, A. K. Geim, R. R. Nair, Nat. Nanotechn. 2017, 12, 546.

[8] B. Brodie, Philos. Trans. R. Soc. London 1859, 149, 249-259.

[9] L. Staudenmaier, Ber. Dtsch. Chem. Ges. 1898, 21, 14811487.

[10] W. S. Hummers, R. E. Offeman, J. Am. Chem. Soc. 1958, 80, 1339-1339.

[11] G. Charpy, Hebd. Séances Acad. Sci 1909, 148, 920-923.

[12] a) J. Chen, B. W. Yao, C. Li, G. Q. Shi, Carbon 2013, 64, 225229; b) R. Muzyka, M. Kwoka, L. Smedowski, N. Diez, G. Gryglewicz, New Carbon Mater. 2017, 32, 15-20; c) J. Chen, Y. Zhang, M. Zhang, B. W. Yao, Y. R. Li, L. Huang, C. Li, G. Q. Shi, Chem. Sci. 2016, 7, 1874-1881; d) X. Y. Yang, X. Y. Zhang, Y. F. Ma, Y. Huang, Y. S. Wang, Y. S. Chen, J. Mater. Chem. 2009, 19, 2710-2714; e) C. G. Liu, Z. N. Yu, D. Neff, A. Zhamu, B. Z. Jang, Nano Lett. 2010, 10, 4863-4868; f) X. L. Li, H. L. Wang, J. T. Robinson, H. Sanchez, G. Diankov, H. J. Dai, J. Am. Chem. Soc. 2009, 131, 15939-15944; g) S. Eigler, M. Enzelberger-Heim, S. Grimm, P. Hofmann, W. Kroener, A. Geworski, C. Dotzer, M. Rockert, J. Xiao, C. Papp, O. Lytken, H. P. Steinruck, P. Muller, A. Hirsch, Adv. Mater. 2013, 25, 3583-3587. 
[13] D. C. Marcano, D. V. Kosynkin, J. M. Berlin, A. Sinitskii, Z. Sun, A. Slesarev, L. B. Alemany, W. Lu, J. M. Tour, ACS Nano 2010, 4, 4806-4814

[14] L. Peng, Z. Xu, Z. Liu, Y. Y. Wei, H. Y. Sun, Z. Li, X. L. Zhao, C. Gao, Nat. Commun. 2015, 6.

[15] S. F. Pei, Q. W. Wei, K. Huang, H. M. Cheng, W. C. Ren, Nat. Commun. 2018, 9

[16] S. Chandra, S. Sahu, P. Pramanik, Mater. Sci. Eng., B 2010 167, 133-136

[17] a) R. Sitko, B. Zawisza, E. Talik, P. Janik, G. Osoba, B. Feist, E. Malick, Anal. Chim. Acta 2014, 834, 22-29; b) R. Sitko, E. Turek, B. Zawisza, E. Malicka, E. Talik, J. Heimann, A. Gagor, B. Feist, R. Wrzalik, Dalton Trans. 2013, 42, 5682-5689; c) B. Zawisza, R. Sitko, E. Malicka, E. Talik, Anal. Methods 2013, 5 6425-6430; d) S. Sahila, L. S. Jayakumari, Polym. Compos 2015, 36, 1-7.

[18] a) W. Gao, L. B. Alemany, L. Ci, P. M. Ajayan, Nat. Chem. 2009, 1, 403-408; b) W. W. Cai, R. D. Piner, F. J. Stadermann, S. Park, M. A. Shaibat, Y. Ishii, D. X. Yang, A. Velamakanni, S. J. An, M. Stoller, J. H. An, D. M. Chen, R. S. Ruoff, Science 2008, 321, 1815-1817.

[19] a) A. Jung, R. Graupner, L. Ley, A. Hirsch, Phys. Status Solidi $B$ 2006, 243, 3217-3220; b) S. Kundu, Y. Wang, W. Xia, M. Muhler, J. Phys. Chem. C 2008, 112, 16869-16878.

[20] A. Fujimoto, Y. Yamada, M. Koinuma, S. Sato, Anal. Chem 2016, 88, 6110-6114.

[21] A. Lerf, H. Y. He, M. Forster, J. Klinowski, J. Phys. Chem. B 1998, 102, 4477-4482.

[22] a) X. Sun, Z. Liu, K. Welsher, J. T. Robinson, A. Goodwin, S. Zaric, H. Dai, Nano Res. 2008, 1, 203-212; b) J. Luo, L. J. Cote, V. C. Tung, A. T. L. Tan, P. E. Goins, J. Wu, J. Huang, J. Am. Chem. Soc. 2010, 132, 17667-17669.

[23] M. Rosillo-Lopez, C. G. Salzmann, Carbon 2016, 106, 56-63.

[24] D. Hadzi, A. Novak, J. Chem. Soc. Faraday Trans. 1955, 51, 1614-1620

[25] C. J. Brooks, G. Eglinton, J. F. Morman, J. Am. Chem. Soc. 1961, 106-\&

[26] M. Rosillo-Lopez, T. J. Lee, M. Bella, M. Hart, C. G. Salzmann, RSC Adv. 2015, 5, 104198-104202.

[27] a) D. Williams, I. Fleming, Spectroscopic Methods in Organic Chemistry, McGraw-Hill Education, UK, 2008; b) M. Acik, G. Lee, C. Mattevi, A. Pirkle, R. M. Wallace, M. Chhowalla, K. Cho, Y. Chabal, J. Phys. Chem. C 2011, 115, 19761-19781.

[28] A. M. Dimiev, L. B. Alemany, J. M. Tour, ACS Nano 2013, 7 , 576-588.

[29] a) R. Beams, L. G. Cancado, L. Novotny, AIP Adv. 2015, 27 ; b) F. Tuinstra, J. L. Koenig, J. Chem. Phys. 1970, 53, 1126-\&; c) J. Maultzsch, S. Reich, C. Thomsen, Phys. Rev. B 2004, 70; d) R. Saito, A. Jorio, A. G. Souza, G. Dresselhaus, M. S. Dresselhaus, M. A. Pimenta, Phys. Rev. Lett. 2002, 88; e) M. A. Pimenta, G. Dresselhaus, M. S. Dresselhaus, L. G.
Cancado, A. Jorio, R. Saito, Phys. Chem. Chem. Phys. 2007, 9, 1276-1291.

[30] a) Z. L. Hu, Y. F. Chen, Q. L. Hou, R. M. Yin, F. S. Liu, H. Chen, New J. Chem. 2012, 36, 1373-1377; b) S. Eigler, A. Dimiev, in Graphene Oxide. Fundamental and Applications, Wiley and Sons, London, 2017; c) H. M. A. Hassan, V. Abdelsayed, A. Khder, K. M. AbouZeid, J. Terner, M. S. ElShall, S. I. Al-Resayes, A. A. El-Azhary, J. Mater. Chem. 2009, 19, 3832-3837; d) X. Y. Xu, T. Wu, F. L. Xia, Y. Li, C. C. Zhang, L. Zhang, M. X. Chen, X. C. Li, Y. Liu, J. P. Gao, J. Power Sources 2014, 266, 282-290; e) G. X. Wang, J. Yang, J. Park, X. L. Gou, B. Wang, H. Liu, J. Yao, J. Phys. Chem. C 2008, 112, 8192-8195; f) S. Stankovich, D. A. Dikin, R. D. Piner, K. A. Kohlhaas, A. Kleinhammes, Y. Jia, Y. Wu, S. T. Nguyen, R. S. Ruoff, Carbon 2007, 45, 1558-1565; g) S. Eigler, C. Dotzer, F. Hof, W. Bauer, A. Hirsch, Chem. A. Eur. J. 2013, 19, 9490-9496

[31] A. Ganguly, S. Sharma, P. Papakonstantinou, J. Hamilton, J. Phys. Chem. C 2011, 115, 17009-17019.

[32] C. A. Amadei, P. Arribas, C. D. Vecitis, Carbon 2018, 133, 398-409.

[33] E. A. Y. Sheng, A. Adriano, C. C. Kiang, Š. Filip, S. Zdeněk, P Martin, Chem. Eur. J. 2013, 19, 12673-12683.

[34] a) I. Y. Jeon, Y. R. Shin, G. J. Sohn, H. J. Choi, S. Y. Bae, J. Mahmood, S. M. Jung, J. M. Seo, M. J. Kim, D. W. Chang, L. M. Dai, J. B. Baek, Proc. Natl. Acad. Sci. U.S.A. 2012, 109, 5588-5593; b) N. Pan, D. B. Guan, Y. T. Yang, Z. L. Huang, R B. Wang, Y. D. Jin, C. Q. Xia, Chem. Eng. J. 2014, 236, 471479; c) A. Y. S. Eng, Z. Sofer, D. Sedmidubsky, M. Pumera, ACS Nano 2017, 11, 1789-1797; d) R. Imani, S. H. Emami, S. Faghihi, J. Nanopart. Res. 2015, 17; e) C. Wu, Y. L. Yuan, Q. He, R. Song, Nanotechnology 2016, 27; f) C. G. Salzmann, V. Nicolosi, M. L. H. Green, J. Mater. Chem. 2010, 20, 314-319; g) F. Ye, C. Feng, J. Jiang, S. Han, Electrochim. Acta 2015, 182, 935-945; h) S. Yu, J. Liu, W. Zhu, Z.-T. Hu, T.-T. Lim, X. Yan, Sci. Rep. 2015, 5; i) Z. Zhong, G. Li, Z. Luo, Z. Liu, Y. Shao, W. He, J. Deng, X. Luo, Anal. Chim. Acta 2015, 888 75-84; j) M. M. Bidgoli, M. Mohsennia, F. A. Boroumand, Bull. Chem. Soc. Jpn. 2015, 88, 684-689; k) S. Zhang, X. Chen, G. Liu, X. Hou, Y. Huang, J. Chen, G. Zhan, C. Li, Electrochim. Acta 2015, 155, 45-53; I) C. Karuppiah, S. Cheemalapati, S. M. Chen, S. Palanisamy, Ionics 2015, 21, 231-238; m) X. Fan, K. Shang, B. Sun, L. Chen, S. Ai, J. Mater. Sci. 2014, 49, 2672-2679; n) G. Wang, X. Tan, Q. Zhou, Y. Liu, M. Wang, L. Yang, Sens. Actuators B Chem. 2014, 190, 730-736; o) O. Jankovsky, M. Novacek, J. Luxa, D. Sedmidubsky, V. Fila, M. Pumera, Z. Sofer, Chem. Eur. J. 2016, 22, 17416-17424; p) C R. Crick, J. Y. Y. Sze, M. Rosillo-Lopez, C. G. Salzmann, J. B. Edel, ACS Appl. Mater. Interfaces 2015, 7, 18188-18194; q) C G. Salzmann, S. A. Llewellyn, G. Tobias, M. A. H. Ward, Y. Huh, M. L. H. Green, Adv. Mater. 2007, 19, 883-887. 


\section{Entry for the Table of Contents}

\section{FULL PAPER}

Making highly carboxylated graphene oxide in a day. Given the widespread use of graphene oxide (GO), a better understanding of the oxidation mechanisms of graphite is highly desirable. Here we present an optimized preparation procedure for making GO by using potassium dichromate as the oxidizing agent. The new GO material is highly carboxylated which will benefit many applications.

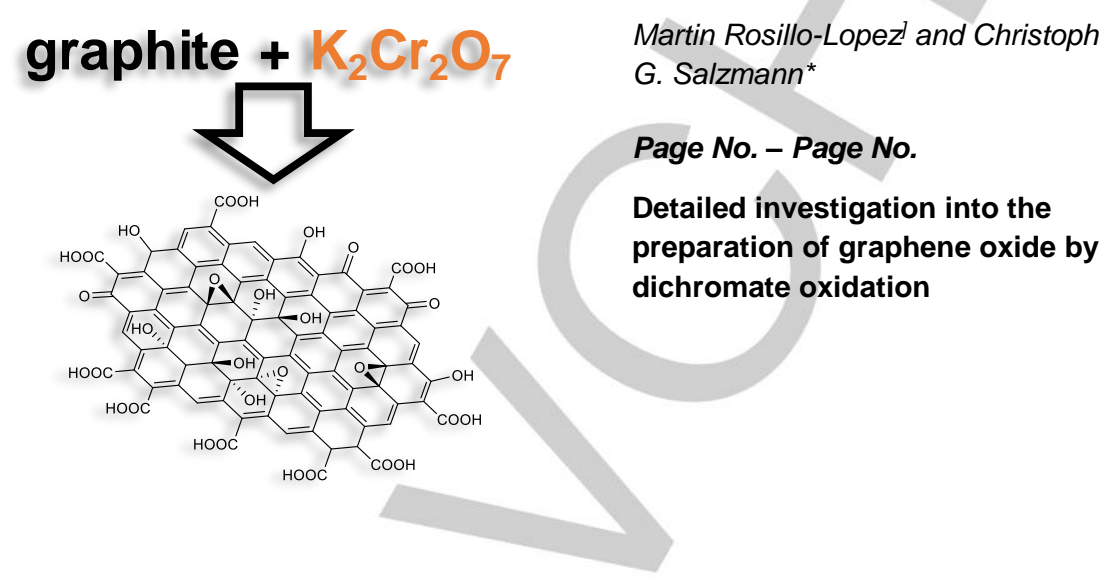

